\title{
Adolescent affective symptoms and mortality
}

\author{
Gemma Archer, Diana Kuh, Matthew Hotopf, Mai Stafford and Marcus Richards
}

\section{Background}

Little is known about the relationship between adolescent affective problems (anxiety and depression) and mortality.

\section{Aims}

To examine whether adolescent affective symptoms are associated with premature mortality, and to assess whether this relationship is independent of other developmental factors.

\section{Method}

Data $(n=3884)$ was from Britain's oldest birth cohort study - the National Survey of Health and Development. Adolescent affective symptoms were rated by teachers at ages 13 and 15 years: scores were summed and classified into three categories: mild or no, moderate and severe symptoms (1st-50th, 51st-90th and 91st-100th percentiles, respectively). Mortality data were obtained from national registry data up to age 68 years. Potential confounders were parental social class, childhood cognition and illness, and adolescent externalising behaviour.

\section{Results}

Over the 53-year follow-up period, $12.2 \%(n=472)$ of study members died. Severe adolescent affective symptoms were associated with an increased rate of mortality compared with those with mild or no symptoms (gender adjusted hazard ratio $1.76,95 \% \mathrm{Cl} 1.33-2.33)$. This association was only partially attenuated after adjustment for potential confounders (fully adjusted hazard ratio $1.61,95 \% \mathrm{Cl} 1.20-2.15)$. There was suggestive evidence of an association across multiple causes of death. Moderate symptoms were not associated with mortality.

\section{Conclusions}

Severe adolescent affective symptoms are associated with an increased rate of premature mortality over a 53-year follow-up period, independent of potential confounders. These findings underscore the importance of early mental health interventions.

\section{Declaration of interest}

None.

\section{Keywords}

Mortality; depression; adolescent; cohort studies; affective disorders.

\section{Copyright and usage}

(C) The Royal College of Psychiatrists 2018. This is an Open Access article, distributed under the terms of the Creative Commons Attribution licence (http://creativecommons.org/licenses/by/ 4.0/), which permits unrestricted re-use, distribution, and reproduction in any medium, provided the original work is properly cited.
Adolescent affective symptoms are associated with a range of negative adult outcomes, including low levels of educational attainment, ${ }^{1,2}$ negative health behaviours ${ }^{3-5}$ and adult mental health problems, ${ }^{6}$ which in turn are associated with excess mortality across multiple causes of death. ${ }^{7-10}$ It is therefore plausible that adolescent affective symptoms are themselves an important risk factor for premature mortality; however, to our knowledge, only two studies have examined this. Lee et al., ${ }^{11}$ demonstrated an association between high adolescent trait anxiety and non-accidental mortality up to age 53 years in the Medical Research Council's National Survey of Health and Development (NSHD; also known as the 1946 British birth cohort study); however, analyses were adjusted for gender only and anxiety was assessed by a single-item measure that classified over $45 \%$ of the sample as a 'case'. Likewise, Jokela et al. $^{12}$ used a relatively weak exposure measure, capturing symptoms that were not a direct measure of internalising disorder. Nevertheless, they found an association between this measure and premature mortality up to age 46 years in the 1958 British birth cohort, although the association was not robust to adjustment for confounders, including childhood social class, cognition and externalising behaviours. We therefore used data from the NSHD when followed to age 68 years, to i. examine whether adolescent affective symptoms (rated in detail by teachers) were associated with all-cause mortality; ii. to assess whether this association was independent of numerous potential confounders, including parental social class, childhood health and cognition, and concurrent mental health problems; and iii. to examine whether associations were observed across multiple causes of death.

\section{Method}

\section{Data}

The NSHD is Britain's oldest birth cohort study. A socially stratified sample of 5362 (2547 female, 2815 male) singleton births were selected for follow-up from all births occurring during a single week in March 1946. The cohort has been followed up 24 times across the life course, most recently at ages 68-69 years. ${ }^{13}$ Participation rates have been high throughout the study, and comparisons between the NSHD and census data have shown that the remaining cohort sample is broadly representative of all nativeborn adults in the general population. ${ }^{14,15}$

Ethical approval was granted by the National Research Ethics Service Committee London Queen Square (14/LO/1073) and by the Scotland A Research Ethics Committee (14/SS/1009). All study members gave signed informed consent.

\section{Mortality}

Mortality data were obtained from study records and linked with National Health Service Central Registry data. Cause of death was coded according to the ICD-9 and ICD-10. ${ }^{16,17}$ We focused on main causes of death: cardiovascular disease (ICD-9 codes 401-454 and ICD-10 codes 110-189), cancers (ICD-9 codes 140-239 and ICD-10 codes C00-C97) and externalising causes (violent, accidental and suicidal deaths; ICD-9 codes 800-994, 1800-1869 and 1880-1999 and ICD-10 codes S00-X99); however, all other causes were identified. Follow-up time was from age 15 years to mortality, until censored owing to emigration $(n=284)$ or to the end of October 2014. 


\section{Adolescent affective symptoms}

Affective symptoms were assessed by teachers, using a forerunner of the Rutter B questionnaire, ${ }^{18}$ which predates the introduction of diagnostic criteria. At ages 13 and 15 years, teachers were asked to rate the study member's behaviour compared with other children in the class, using a three-category response scale: more than, the same or less than other children. The questionnaires were subjected to exploratory factor analysis, which identified three distinct factors relating to emotional problems, externalising behaviour (conduct problems) and self-organisation. ${ }^{19,20}$ Ten items were indicators of emotional problems: anxious, always tired and washed out, frightened of rough games, extremely fearful, avoids attention, usually gloomy and sad, timid child, unable to make friends, diffident about competing and unduly miserable or worried about criticism; model fit indices of measurement invariance indicated excellent fit, suggesting adequate reliability. ${ }^{20}$ The factor scores of Xu et al. ${ }^{20}$ for emotional problems were standardised with $z$-scores at ages 13 and 15 years, and then summed to create a single measure of adolescent affective symptoms.

We generated a three-category ordinal variable based on previously used cut-off points: ${ }^{2,19}$ study members between the 1st and 50 th percentiles were classified as having mild or no symptoms, those between the 50th and 90th percentiles were classified as having moderate symptoms and those between the 91st and 100th percentiles were classified as having severe symptoms. The cut-off point for severe symptoms is in keeping with 12-month prevalence rates for adolescent anxiety and depressive disorders reported in samples in Europe and the USA ${ }^{21}(6.9-9.5 \%$ for anxiety and $2.1-3.4 \%$ for depressive disorders).

\section{Confounding factors}

Potential confounders were identified a priori as childhood social, psychological and physical health factors associated with lifetime mental health and mortality in other studies, including the NSHD. ${ }^{22-24}$ These were prospectively measured childhood social class (based on the occupation of the study member's father at age 11 years, or if this was unknown, at age 4 or 15 years, and coded into six groups according to the Registrar-General's classification), childhood cognition at age 8 years (derived from tests designed by the National Foundation for Education Research and described in detail elsewhere ${ }^{25}$ ), teacher-rated adolescent externalising behaviour at ages 13-15 years (derived from factor analysis by $\mathrm{Xu}$ et $a .^{20}$ ), birth weight (in kilograms, obtained from birth records), childhood sickness absence (0-4 weeks, 4-10 weeks and 10+ weeks, obtained from school records spanning ages 6-12 years) and childhood hospital admission at ages $0-5$ years, $6-10$ years and $11-15$ years (obtained from parental interviews, school attendance records, medical examinations and hospital in-patient records). Schizophrenia was ascertained by questionnaire, interview, and hospital and general practitioner contact data up to age 43 years. ${ }^{26}$ Health behaviours and other adult psychiatric or psychological problems ${ }^{27}$ were not included as potential confounders because we considered these variables to be potential mediators on the causal pathway.

\section{Analyses}

We used Kaplan-Meier graphs to compare the survival probability of those with mild or no, moderate and severe affective symptoms over the follow-up period, and tested the equality of survival curves with a log-rank test. Cox proportional hazards models were used to investigate the relationship between adolescent affective symptoms and all-cause mortality rates. Models were first adjusted for gender. The gender-adjusted hazard ratios for affective symptoms were then adjusted for each potential confounder in turn, with childhood sickness absence and hospital admissions grouped to represent physical health. A further model included all variables. The analyses were repeated using competing-risks analyses, to examine whether associations between affective symptoms and mortality were observed across different causes of death (cardiovascular, cancer, externalising and all other causes).

Gender interactions were tested using joint Wald tests; however, there was no evidence that associations differed in males and females $(P=0.86$ for all-cause mortality and $P=0.19-0.54$ for cause-specific mortality).

The proportional hazards assumption was checked by including an interaction term between log-time and affective symptoms. Associations were robust to sensitivity analyses, which included the use of a less stringent cut-off point to classify severe symptoms (84th-100th percentile) (see supplementary Table 1, available at https://doi.org/10.1192/bjp.2018.90), and the exclusion of people with schizophrenia $(n=24)$ to examine whether associations were driven by concurrent mental disorder.

\section{Sample}

Eligible participants included all those who had complete data on affective symptoms at ages 13 and 15 years and who were linked with the National Health Service Central Register. Of the original birth cohort $(n=5362), 250$ died and 838 refused participation in the study, emigrated or were unable to be traced before age 15 years. A further 342 had missing data on affective symptoms at ages $13-15$ years, and 48 had non-linked mortality data, leaving 3884 study members in the analytical sample. To minimise data loss, multiple imputation with chained equations was used to impute missing data on the following covariates: birth weight ( $n=17,0.4 \%)$, childhood social class $(n=55,1.4 \%)$, childhood cognition $(n=165,4.2 \%)$ and childhood sickness absence $(n=647$, $16.7 \%){ }^{28,29}$ The imputation analyses contained all study variables, including factors that have been shown to predict non-response in the NSHD, such as manual social class and poor childhood cognition. ${ }^{14}$ Analyses were run across 20 imputed data-sets. On visual inspection, the imputed results were very similar to those using observed values (see supplementary Table 2 for non-imputed results, and supplementary Fig. 1 for fully adjusted survival curves based on non-imputed data; the Kaplan-Meier function is not currently supported for imputed data). All analyses were carried out in STATA version 13.1.

\section{Results}

Table 1 shows the characteristics of the original (non-imputed) data and imputed study sample were similar. Mean follow-up for mortality after age 15 years was 48.8 years (range 3.4-53.0), with a total of 189609 person-years and 472 deaths. Males had a slightly higher mortality rate than females (2.75 compared with 2.21 per 1000 person-years, respectively). For both genders, the most common cause of death was cancer, followed by cardiovascular disease and externalising causes (violent, accidental or suicidal deaths). All other causes of death comprised diseases of the respiratory system (33.0\%), digestive system (23.0\%), nervous system (16.5\%) and all other miscellaneous causes (27.5\%). Affective symptoms were more commonly reported in females than males, with $10.5 \%$ of females and $7.6 \%$ of males rated as having severe symptoms.

Fig. 1 shows that study members rated with severe adolescent affective symptoms had the lowest survival probability throughout the follow-up period, followed by those with moderate and those with mild or no symptoms (the log-rank test showed that there was a difference in survival curves: $\left.\chi^{2}(2)=14.1, P<0.001\right)$. Markedly, the 


\begin{tabular}{|c|c|c|c|c|c|}
\hline & \multicolumn{2}{|c|}{ Non-imputed } & \multicolumn{3}{|c|}{ Imputed } \\
\hline & $N$ & All, \% & All, \% & Males, \% & Females, \% \\
\hline \multicolumn{6}{|l|}{ Mortality } \\
\hline All-cause & 472 & 12.2 & 12.2 & 13.3 & 10.9 \\
\hline \multicolumn{6}{|l|}{ Cause-specific mortality } \\
\hline Cancer & 206 & 43.6 & 43.6 & 41.0 & 47.1 \\
\hline Cardiovascular disease & 117 & 24.8 & 24.8 & 26.5 & 22.6 \\
\hline Externalising causes & 58 & 12.3 & 12.3 & 14.2 & 9.8 \\
\hline Other $^{\mathrm{a}}$ & 91 & 19.3 & 19.3 & 18.3 & 20.6 \\
\hline \multicolumn{6}{|l|}{ Affective symptoms } \\
\hline None/mild & 1942 & 50.0 & 50.0 & 53.9 & 45.8 \\
\hline Moderate & 1592 & 41.0 & 41.0 & 38.4 & 43.7 \\
\hline Severe & 350 & 9.0 & 9.0 & 7.6 & 10.5 \\
\hline \multicolumn{6}{|l|}{ Childhood social class } \\
\hline Professional & 220 & 5.8 & 5.7 & 5.7 & 5.7 \\
\hline Intermediate & 703 & 18.4 & 18.3 & 19.2 & 17.4 \\
\hline Skilled, non-manual & 572 & 14.9 & 14.9 & 14.6 & 15.2 \\
\hline Skilled, manual & 1314 & 34.3 & 34.3 & 34.0 & 34.7 \\
\hline Partly skilled & 760 & 19.9 & 19.9 & 19.4 & 20.4 \\
\hline Unskilled & 260 & 6.8 & 6.8 & 7.1 & 6.5 \\
\hline \multicolumn{6}{|l|}{ Birth weight, $\mathrm{kg}$} \\
\hline Mean (s.d.) & 3867 & $3.40(0.51)$ & $3.40(0.51)$ & $3.48(0.52)$ & $3.32(0.50)$ \\
\hline \multicolumn{6}{|c|}{ Childhood sickness absence, weeks } \\
\hline $0-4$ & 1680 & 51.9 & 52.7 & 53.5 & 51.8 \\
\hline $4-10$ & 1183 & 36.6 & 36.4 & 36.3 & 36.5 \\
\hline $10+$ & 374 & 11.6 & 10.9 & 10.2 & 11.7 \\
\hline \multicolumn{6}{|c|}{ Childhood hospital admissions, years } \\
\hline $0-5$ & 255 & 6.6 & 6.6 & 7.1 & 5.9 \\
\hline $6-10$ & 519 & 13.4 & 13.4 & 14.3 & 12.3 \\
\hline $11-15$ & 210 & 5.4 & 5.4 & 6.1 & 4.7 \\
\hline
\end{tabular}

survival curve for severe symptoms continued to diverge for the duration of the follow-up period; the proportional hazards assumption was not violated $(P=0.58)$. By age 68 years, $18.6 \%$ of the severe group had died compared with $10.8 \%$ in the mild or no problem group.

Table 2 shows the association between adolescent affective symptoms and all-cause mortality, adjusted for potential confounders. After adjustment for gender, severe affective symptoms were associated with a higher rate of mortality compared with those with mild or no symptoms (hazard ratio 1.76, 95\% CI 1.33-2.33). Childhood social class, cognition, birth weight, sickness absence and hospital admissions all slightly attenuated the association. Adolescent externalising behaviour was a weak negative confounder, whereas birth weight had no explanatory role. After adjustment for all potential confounders, the association remained but was partially

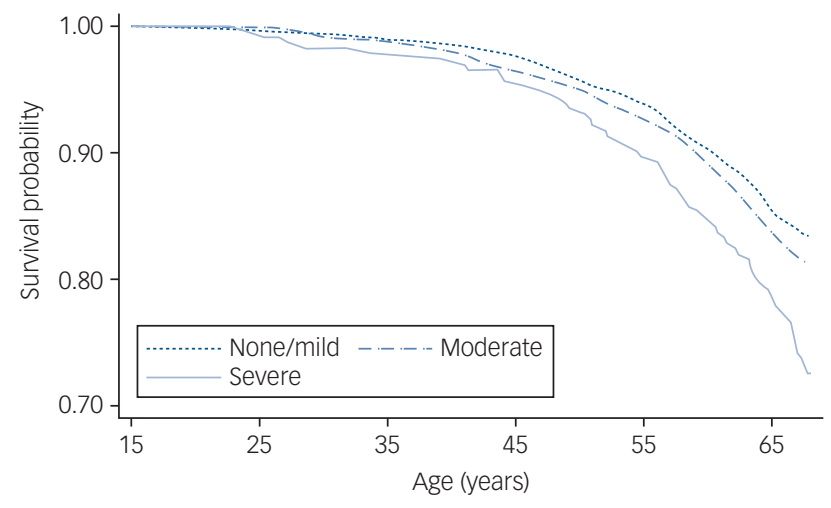

Fig. 1 Gender-adjusted Kaplan-Meier survival curves for all-cause mortality by adolescent affective symptoms, based on 472 deaths $(n=3884)$ attenuated (hazard ratio $1.61,95 \%$ CI 1.20-2.15). The exclusion of people with schizophrenia did not alter the results (hazard ratio $1.58,95 \%$ CI 1.18-2.13). The hazard ratios for moderate compared with mild or no symptoms were only slightly raised across all analyses and these associations were not statistically significant.

Table 3 shows associations between affective symptoms and mortality, by cause of death. After adjustment for gender, severe affective symptoms were associated with an increased rate of cardiovascular mortality (subdistribution hazard ratio 1.92, 95\% CI 1.11-3.32) and other causes of death (subdistribution hazard ratio 2.65, 95\% CI 1.47-4.80), and showed a borderline association with cancer mortality (subdistribution hazard ratio 1.52, 95\% CI 1.00-2.31), compared with those with mild or no symptoms. There was no evidence of an association between severe affective symptoms and externalising causes of death; however, reliable estimates could not be generated in this subgroup as there were only four from externalising causes deaths (one of which was suicide) among those with severe symptoms.

After adjustment for all potential confounders, an association remained between severe affective symptoms and other causes of death (subdistribution hazard ratio 2.03, 95\% CI 1.07-3.85), but all other associations were attenuated to non-significance. The gender-adjusted subdistribution hazard ratios for cardiovascular mortality were attenuated by childhood social class (subdistribution hazard ratio $1.76,95 \%$ CI 1.01-3.06), cognition (subdistribution hazard ratio $1.61,95 \%$ CI $0.92-2.82$ ), and childhood sickness absence and hospital admissions (subdistribution hazard ratio 1.82 , 95\% CI 1.06-3.16), whereas the gender-adjusted subdistribution hazard ratios for other causes were attenuated only by cognition (subdistribution hazard ratio 2.31, 95\% CI 1.26-4.24) and childhood sickness absence and hospital admissions (subdistribution hazard ratio 2.21, 95\% CI 1.20-4.05) (see supplementary Table 3). Estimates for cancer and externalising causes did not change with these adjustments (not shown). 


\begin{tabular}{|c|c|c|c|}
\hline Model & $\begin{array}{l}\text { Affective } \\
\text { symptoms }\end{array}$ & $\begin{array}{l}\text { Hazard ratio } \\
(95 \% \mathrm{Cl})\end{array}$ & $\begin{array}{l}\text { Test for linear } \\
\text { trend ( } P \text { value) }\end{array}$ \\
\hline Adjusted for gender & $\begin{array}{l}\text { None/mild } \\
\text { Moderate } \\
\text { Severe }\end{array}$ & $\begin{array}{l}1.0 \\
1.15(0.95-1.40) \\
1.76(1.33-2.33)^{\star *}\end{array}$ & $<0.001$ \\
\hline Adjusted for gender and childhood social class & $\begin{array}{l}\text { None/mild } \\
\text { Moderate } \\
\text { Severe }\end{array}$ & $\begin{array}{l}1.0 \\
1.12(0.92-1.37) \\
1.67(1.26-2.21)^{\star \star}\end{array}$ & $<0.01$ \\
\hline Adjusted for gender and cognition at age 8 years & $\begin{array}{l}\text { None/mild } \\
\text { Moderate } \\
\text { Severe }\end{array}$ & $\begin{array}{l}1.0 \\
1.12(0.92-1.37) \\
1.63(1.23-2.17)^{\star}\end{array}$ & $<0.01$ \\
\hline Adjusted for gender and externalising behaviour at age 13-15 years & $\begin{array}{l}\text { None/mild } \\
\text { Moderate } \\
\text { Severe }\end{array}$ & $\begin{array}{l}1.0 \\
1.18(0.97-1.43) \\
1.81(1.37-2.40)^{\star *}\end{array}$ & $<0.001$ \\
\hline Adjusted for gender and birth weight & $\begin{array}{l}\text { None/mild } \\
\text { Moderate } \\
\text { Severe }\end{array}$ & $\begin{array}{l}1.0 \\
1.15(0.95-1.40) \\
1.76(1.33-2.33)^{\star * *}\end{array}$ & $<0.001$ \\
\hline $\begin{array}{l}\text { Adjusted for gender, sickness absence at age 6-10 years and hospital } \\
\text { admissions at ages 0-6, 6-10 and 11-15 years }\end{array}$ & $\begin{array}{l}\text { None/mild } \\
\text { Moderate } \\
\text { Severe }\end{array}$ & $\begin{array}{l}1.0 \\
1.14(0.94-1.39) \\
1.64(1.23-2.18)^{\star}\end{array}$ & $<0.01$ \\
\hline Adjusted for all & $\begin{array}{l}\text { None/mild } \\
\text { Moderate } \\
\text { Severe }\end{array}$ & $\begin{array}{l}1.0 \\
1.13(0.93-1.37) \\
1.61(1.20-2.15)^{*}\end{array}$ & $<0.01$ \\
\hline
\end{tabular}

Table 3 Subdistribution hazard ratios for the association between affective symptoms at age 13-15 years and cause-specific mortality (15-67 years), based on 20 imputations $(n=3884)$.

\begin{tabular}{|c|c|c|c|}
\hline \multirow[b]{2}{*}{ Cause of death } & \multirow[b]{2}{*}{ Affective symptoms } & \multicolumn{2}{|c|}{ Subdistribution hazard ratio $(95 \% \mathrm{Cl})$} \\
\hline & & Gender adjusted & Fully adjusted $^{a}$ \\
\hline \multirow[t]{3}{*}{ Cancer (206 deaths) } & None/mild & 1.0 & 1.0 \\
\hline & Moderate & $0.94(0.70-1.26)$ & $0.94(0.69-1.26)$ \\
\hline & Severe & $1.52(1.00-2.31)$ & $1.49(0.97-2.28)$ \\
\hline \multirow[t]{3}{*}{ Cardiovascular disease (117 deaths) } & None/mild & 1.0 & 1.0 \\
\hline & Moderate & $1.24(0.83-1.84)$ & $1.16(0.79-1.72)$ \\
\hline & Severe & $1.92(1.11-3.32)^{\star}$ & $1.65(0.93-2.91)$ \\
\hline \multirow[t]{3}{*}{ Externalising causes (58 deaths) } & None/mild & 1.0 & 1.0 \\
\hline & Moderate & $1.25(0.73-2.14)$ & $1.28(0.74-2.23)$ \\
\hline & Severe & $0.85(0.29-2.47)$ & $0.89(0.31-2.56)$ \\
\hline \multirow[t]{3}{*}{ Other $^{\mathrm{b}}$ (91 deaths) } & None/mild & 1.0 & 1.0 \\
\hline & Moderate & $1.55(0.99-2.44)$ & $1.44(0.91-2.27)$ \\
\hline & Severe & $2.65(1.47-4.80)^{\star}$ & $2.03(1.07-3.85)^{*}$ \\
\hline \multicolumn{4}{|c|}{$\begin{array}{l}\text { a. Adjusted for gender, childhood social class, cognition at age } 8 \text { years, externalising behaviour at age } 13-15 \text { years, birth weight, sickness absence at age } 6-10 \text { years and hospital admissions } \\
\text { at ages } 0-6,6-10 \text { and } 11-15 \text { years. } \\
\text { b. Diseases of the respiratory system (33\%), digestive system (23\%), nervous system (16.5\%) and all other causes (27.5\%). } \\
\text { * } P<0.05 \text {. }\end{array}$} \\
\hline
\end{tabular}

\section{Discussion}

In this large, UK population-based cohort, we found that severe adolescent affective symptoms were associated with a $61 \%$ increase in premature mortality over a 53 -year follow-up period compared with those who had mild or no symptoms (hazard ratio $1.61,95 \%$ CI $1.20-2.15, P<0.05$; equivalent to an additional 66 deaths per 1000 people). Notably, this relationship persisted for the duration of the follow-up period and was independent of a wide range of potential confounders, including parental social class, childhood hospital admissions, sickness absence and cognition, and adolescent externalising behaviour. There was also evidence to suggest that associations were observed across multiple causes of death.

\section{Comparison with other studies}

Our study is the most comprehensive examination of the relationship between adolescent affective symptoms and premature mortality to date; we build on existing studies by extending mortality follow-up by at least 15 years, providing more rigorous control for potential confounding factors and using a stronger measure of affective problems. ${ }^{11,12}$ The findings are largely consistent with two previous studies examining the association between adolescent affective symptoms and mortality. Lee et al. ${ }^{11}$ and Jokela et al. ${ }^{12}$ both demonstrated gender-adjusted associations between adolescent affective symptoms and greater risk of premature mortality in population-based samples over a long period of follow-up. Jokela et al. ${ }^{12}$ found that associations were attenuated to non-significance after adjustment for adolescent externalising behaviour and other covariates such as cognitive ability and father's social class. This could be partly attributed to the study's measure of exposure (the 'under-reaction' dimension of the Bristol SocialAdjustment Guide ${ }^{30}$ ), which the author's note, could be considered related to, but not a direct measure of internalising. Furthermore, our study extends the follow-up period of Jokela et al. ${ }^{12}$ by almost 20 years, which could be important because causes of death vary by age; for instance, deaths from cancer and 
cardiovascular disease are more common at older ages compared with deaths from externalising causes. ${ }^{31}$ In the NSHD, Lee et al. ${ }^{11}$ found that high adolescent anxiety was associated with higher rate of non-accidental mortality after age 25 years, but a lower rate of accidental mortality before age 25 years. In keeping with Jokela et al., ${ }^{12}$ we found no evidence of a protective effect of affective symptoms in our analyses. Lee et al. ${ }^{11}$ used a singleitem measure of anxiety that classified over $50 \%$ of study members as a 'case', which may help to explain this discrepancy.

There is substantial continuity between depression experienced in adolescence and depression in adulthood; ${ }^{6,32}$ therefore, it is likely that the observed associations are, at least in part, a result of an accumulation effect. Equally, however, affective symptoms in adolescence may be especially detrimental because of their effect on educational attainment ${ }^{1,2}$ and subsequent negative social, psychological and behavioural factors. ${ }^{1-3,6}$ Because these factors are wide-ranging, it is understandable that we observed associations with mortality across several different causes of death. In particular, we found associations with deaths from cancers and cardiovascular disease after adjustment for gender; although the strongest associations were with deaths from other causes (predominantly diseases of the respiratory, digestive and nervous systems), which held after full adjustment for confounders. We did not have adequate power to examine the association between affective symptoms and deaths from other causes in more detail; however, the results are consistent with existing studies that have used mixed-age community-based samples, and have shown that depression and anxiety disorders are associated with multiple causes of death, including diseases of the metabolism, respiratory and nervous systems, and external causes such as accidents and suicide. ${ }^{9,33}$

A key criticism of existing literature examining affective symptoms and mortality is a general failure to account for potential confounding with concurrent physical and mental health. ${ }^{34}$ Schizophrenia and adolescent externalising behaviour have demonstrated strong associations with premature mortality in the $\mathrm{NSHD}^{23}$ and elsewhere; ${ }^{12,35}$ however, our results were not attenuated by controlling for externalising behaviour, nor were they explained by excluding people with schizophrenia. Likewise, we found little evidence that the associations between affective symptoms and mortality were due to confounding with poor physical health, as controlling for factors relating to poor physical health in childhood, including hospital admissions, sickness absence and birth weight, did little to attenuate associations.

\section{Strengths and limitations}

Methodological strengths of our study include an exceptionally long follow-up period and prospectively obtained data on a wide range of potential confounding variables, although the possibility of residual confounding cannot be excluded. Loss to follow-up was particularly low as there were only 49 study members with non-linked mortality data, and missing covariate data were handled with multiple imputation. Our measure of affective symptoms strongly predicts adult mental disorder in the NSHD, ${ }^{6}$ suggesting good construct validity; furthermore, teacher-rated data have been shown to better predict psychiatric disorder than self-report data in other measures of child and adolescent emotional problems, as shown by the Strength and Difficulties Questionnaire. $^{36}$

Limitations of this study include low power to detect small effects, and so it was difficult to draw strong conclusions about moderate symptoms and estimates regarding cause-specific mortality. Also, the generalisability of the study may be limited because of differences in social and health challenges faced by study members compared with today's children; for example, study members grew up in a post-war economy and in the era of serious childhood diseases such as polio and measles, which have since been effectively eradicated. Likewise, there have been considerable improvements in mental health awareness and treatment since the 1960s, which may buffer the effect of mental health problems on educational and behavioural outcomes associated with mortality; nevertheless, access to adolescent mental health services remains poor, with only around a quarter of young people in the UK getting the help they need. ${ }^{37}$

In conclusion, we have shown that severe adolescent affective symptoms are associated with premature mortality irrespective of a range of potential confounders, including childhood social disadvantage, childhood illness and other mental health problems. Remarkably, the effect of severe adolescent affective symptoms on mortality persisted for the duration of follow-up - over 50 years; however further research is needed to elucidate the role of accumulation and potential mediating mechanisms. There was suggestive evidence of an association between affective symptoms and mortality across multiple causes of death, especially other causes, which warrants more detailed investigation with larger samples as these causes are rare. Given the prevalence of adolescent affective disorder, our findings highlight the importance of early intervention and treatment to prevent associated declines in mental and physical health, and subsequent early mortality. Early identification could be facilitated by routine assessment of mental health during medical check-ups or at school, and enhanced mental health training for health and education professionals.

Gemma Archer, PhD, Diana Kuh, PhD, Medical Research Council Unit for Lifelong Health and Ageing, University College London, UK; Matthew Hotopf, PhD, Institute of Psychiatry, Psychology \& Neuroscience, King's College London, UK, and South London and Maudsley NHS Foundation Trust, UK; Mai Stafford, PhD, Marcus Richards, PhD, Medical Research Council Unit for Lifelong Health and Ageing, University College London, UK

Correspondence: Gemma Archer, PhD, MRC Unit for Lifelong Health and Ageing at UCL, University College London, 33 Bedford Place, London, WC1B 5JU, UK. Email: g.archer@ucl. ac.uk

First received 21 Dec 2017, final revision 3 Apr 2018, accepted 8 Apr 2018

\section{Supplementary material}

Supplementary material is available online at https://doi.org/10.1192/bjp.2018.90.

\section{Funding}

G.A. is supported by an Economic and Social Research Council research studentship. M.S. is supported by the UK Medical Research Council (unit programme number MC_UU_12019/1) supported by the UK Medical Research Council (unit programme number MC_UU_12019/1),
D.K. is funded by the UK Medical Research Council (unit programme numbers MC UU 12019/ 1 and 12019/4) and M.R. is funded by the UK Medical Research Council (unit programme numbers MC UU 12019/1 and 12019/3). This paper represents independent research part-funded by the National Institute for Health Research (NIHR) Biomedical Research Centre at South London and Maudsley NHS Foundation Trust and King's College London. The views expressed are those of the authors and not necessarily those of the NHS, the NHHR or the Department of Health. The of the authors and not necessarly those of the NHS, the NiHR or the Department of Health. The funders had no role in study design, analysis, interpretation of data, writing of the report, or the decision to submit the article for publication.

\section{References}

1 Lacey RE, Kumari M, McMunn A. Parental separation in childhood and adult inflammation: the importance of material and psychosocial pathways. Psychoneuroendocrinology 2013; 38: 2476-84.

2 Richards M, Abbott RA. Childhood Mental Health and Life Chances in Post-war Britain: Insights from Three National Birth Cohort Studies. Centre for Mental Health, 2009.

3 Breslau N, Peterson EL, Schultz LR, Chilcoat HD, Andreski P. Major depression and stages of smoking: a longitudinal investigation. Arch Gen Psychiatry 1998; 55: 161-6. 
4 Fuller-Thomson E, Filippelli J, Lue-Crisostomo CA. Gender-specific association between childhood adversities and smoking in adulthood: findings from a population-based study. Public Health 2013; 127: 449-60.

5 Knüppel A, Shipley MJ, Llewellyn $\mathrm{CH}$, Brunner EJ. Sugar intake from sweet food and beverages, common mental disorder and depression: prospective findings from the Whitehall II study. Sci Rep 2017; 7: 6287.

6 Colman I, Wadsworth MEJ, Croudace TJ, Jones PB. Forty-year psychiatric outcomes following assessment for internalizing disorder in adolescence. Am J Psychiatry 2007; 164: 126-33.

7 Doll R, Peto R, Boreham J, Sutherland I. Mortality in relation to smoking: 50 years' observations on male British doctors. BMJ 2004; 328: 1519.

8 Kant AK, Leitzmann MF, Park Y, Hollenbeck A, Schatzkin A. Patterns of recommended dietary behaviors predict subsequent risk of mortality in a large cohort of men and women in the United States. J Nutr 2009; 139: 1374-80.

9 Mykletun A, Bjerkeset O, Dewey M, Prince M, Overland S, Stewart R. Anxiety, depression, and cause-specific mortality: the HUNT study. Psychosom Med 2007; 69: 323-31.

10 Paffenbarger Jr RS, Hyde RT, Wing AL, Lee I-M, Jung DL, Kampert JB. The association of changes in physical-activity level and other lifestyle characteristics with mortality among men. N Engl J Med 1993; 328: 538-45.

11 Lee WE, Wadsworth MEJ, Hotopf M. The protective role of trait anxiety: a longitudinal cohort study. Psychol Med 2006; 36: 345-51.

12 Jokela M, Ferrie J, Kivimäki M. Childhood problem behaviors and death by midlife: the British National Child Development Study. J Am Acad Child Adolesc Psychiatry 2009; 48: 19-24.

13 Kuh D, Wong A, Shah I, Moore A, Popham M, Curran P, et al. The MRC National Survey of Health and Development reaches age 70: maintaining participation at older ages in a birth cohort study. Eur J Epidemiol 2016; 31: 1135-47.

14 Stafford M, Black S, Shah I, Hardy R, Pierce M, Richards M, et al. Using a birth cohort to study ageing: representativeness and response rates in the Nationa Survey of Health and Development. Eur J Ageing 2013; 10: 145-57.

15 Wadsworth ME, Mann SL, Rodgers B, Kuh DJ, Hilder WS, Yusuf EJ. Loss and representativeness in a 43 year follow up of a national birth cohort. J Epidemiol Community Health 1992; 46: 300-4

16 World Health Organization. ICD-9-CM: International Classification of Diseases, 9th Revision: Clinical Modification. World Health Organization, 1998.

17 World Health Organization. ICD-10: International statistical classification of diseases and related health problems. World Health Organization, 2004

18 Rutter M. A children's behaviour questionnaire for completion by teachers: preliminary findings. J Child Psychol Psychiatry 1967; 8: 1-11.

19 Colman I, Ploubidis GB, Wadsworth ME, Jones PB, Croudace TJ. A longitudinal typology of symptoms of depression and anxiety over the life course. Biol Psychiatry 2007; 62: 1265-71.

20 Xu MK, Jones PB, Barnett JH, Gaysina D, Kuh D, Croudace TJ, et al. Adolescent self-organization predicts midlife memory in a prospective birth cohort study. Psychol Aging 2013; 28: 958-68.

21 Merikangas KR, Nakamura EF, Kessler RC. Epidemiology of mental disorders in children and adolescents. Dialogues Clin Neurosci 2009; 11: 7-20.
22 Kuh D, Richards M, Hardy R, Butterworth S, Wadsworth ME. Childhood cognitive ability and deaths up until middle age: a post-war birth cohort study. Int $J$ Epidemiol 2004; 33: 408-13

23 Maughan B, Stafford M, Shah I, Kuh D. Adolescent conduct problems and premature mortality: follow-up to age 65 years in a national birth cohort. Psychol Med 2014; 44: 1077.

24 Kuh D, Hardy R, Langenberg C, Richards M, Wadsworth ME. Mortality in adults aged 26-54 years related to socioeconomic conditions in childhood and adulthood: post war birth cohort study. BMJ 2002; 325: 1076-80.

25 Richards M, Shipley B, Fuhrer R, Wadsworth ME. Cognitive ability in childhood and cognitive decline in mid-life: Iongitudinal birth cohort study. BMJ 2004; 328: 552 .

26 Jones $\mathrm{P}$, Murray $\mathrm{R}$, Jones $\mathrm{P}$, Rodgers $\mathrm{B}$, Marmot $\mathrm{M}$. Child developmental risk factors for adult schizophrenia in the British 1946 birth cohort. Lancet 1994; 344: 1398-402.

27 Henderson M, Hotopf M, Shah I, Hayes RD, Kuh D. Psychiatric disorder in early adulthood and risk of premature mortality in the 1946 British birth cohort. BMC Psychiatry 2011; 11: 37

28 Carpenter J, Kenward M. Multiple Imputation and its Application. John Wiley \& Sons, 2012.

29 Little RJ, Rubin DB. Statistical Analysis with Missing Data. John Wiley \& Sons, 2014.

30 Stott D. The Social-Adjustment of Children: Manual to the Bristol SocialAdjustment Guides. University of London Press, 1963.

31 Centers for Disease Control and Prevention (CDC). Ten Leading Causes of Death and Injury. CDC, 2015 (https://www.cdc.gov/injury/wisqars/LeadingCauses. $\mathrm{html})$.

32 Harrington R, Fudge $\mathrm{H}$, Rutter M, Pickles A, Hill J. Adult outcomes of childhood and adolescent depression: I. Psychiatric status. Arch Gen Psychiatry 1990; 47: 465-73.

33 Joukamaa M, Heliövaara M, Knekt $\mathrm{P}$, Aromaa A, Raitasalo R, Lehtinen V. Mental disorders and cause-specific mortality. Br J Psychiatry 2001; 179: 498-502.

34 Miloyan B, Fried E. A reassessment of the relationship between depression and all-cause mortality in 3,604,005 participants from 293 studies. World Psychiatry 2017; 16: 219-20.

35 Tsuang MT, Woolson RF. Excess mortality in schizophrenia and affective disorders: do suicides and accidental deaths solely account for this excess? Arch Gen Psychiatry 1978; 35: 1181-5

36 Goodman R. Using the Strengths and Difficulties Questionnaire (SDQ) to screen for child psychiatric disorders in a community sample. Br J Psychiatry 2000; 177: 534-9.

37 Khan L. Missed Opportunities: A Review of the Recent Evidence into Children and Young People's Mental Health. Centre for Mental Health, 2016. 